\title{
SEMIGROUPS OF CONSTANT MAPS
}

\author{
BORIS M. SCHEIN
}

(Received 17 October 1983)

Communicated by T. E. Hall

\begin{abstract}
In this paper "a map" denotes an arbitrary (everywhere defined, or partial, or even multi-valued) mapping. A map is constant if any two elements belonging to its domain have precisely the same images under this map. We characterize those semigroups which can be isomorphic to semigroups of constant maps or to involuted semigroups of constant maps.
\end{abstract}

1980 Mathematics subject classification (Amer. Math. Soc.): primary 20 M 20; secondary 04 A 05 . Keywords and phrases: semigroup, involuted semigroup, semigroup of binary relations, constant mappings.

A map of a set $A$ into itself is any subset $m$ of $A \times A$ (i.e. any binary relation on $A)$. If $a \in A$, then $m\langle a\rangle=\left\{a_{1} \in A:\left(a, a_{1}\right) \in m\right\}$ is the set of all images of $a$ under $m$. A map is called a partial transformation of $A$ whenever $|m\langle a\rangle| \leqslant 1$ for all $a \in A$. The set $\{a: m\langle a\rangle \neq \varnothing\}$ is the first projection (or the domain) of the map $m$; we denote it as $\operatorname{pr}_{1} m$. If $\operatorname{pr}_{1} m=A$, the map $m$ is called total. If $m$ is a map, then $m^{-1}=\left\{\left(a_{1}, a_{2}\right):\left(a_{2}, a_{1}\right) \in m\right\}$ is called the converse map. Its domain $\operatorname{pr}_{1} m^{-1}$ is also denoted as $\operatorname{pr}_{2} m$ and is called the second projection (or the range) of $m$. If $\operatorname{pr}_{2} m=A$, then $m$ is called surjective.

If $m_{1}$ and $m_{2}$ are maps then $m_{2} \circ m_{1}$ is their composite map:

$$
\left(a_{1}, a_{2}\right) \in m_{2} \circ m_{1} \Leftrightarrow(\exists a)\left[\left(a_{1}, a\right) \in m_{1} \text { and }\left(a, a_{2}\right) \in m_{2}\right] \text {. }
$$

If $B$ is a subset of $A$ (i.e. $B \subset A$, which does not preclude $B=A$ ), then $m(B)=\bigcup\{m\langle a\rangle: a \in B\}$ is the set of all images under $m$ of all elements of $B$. In particular, $m(A)=\mathrm{pr}_{2} m$. It is clear that $m_{2} \circ m_{1}(B)=m_{2}\left(m_{1}(B)\right)$. In particular, $m_{2} \circ m_{1}\langle a\rangle=m_{2}\left(m_{1}\langle a\rangle\right)$.

This research was supported in part by dual funding from NSF/EPSCOR Grant ISP 8011447 and the State of Arkansas.

(C) 1985 Australian Mathematical Society 0263-6115/85\$A2.00+0.00 
We are writing maps as left operators. The last equality explains why the product (composition) of two maps, $m_{1}$ and $m_{2}$, is written from right to left, as $m_{2} \circ m_{1}$ rather than $m_{1} \circ m_{2}$.

The set $\mathscr{B}_{A}$ of all maps on a set $A$ forms a semigroup under composition $\circ$. If $S$ is a semigroup, a mapping $h: S \rightarrow \mathscr{B}_{A}$ is a homomorphism whenever $h\left(s_{1} s_{2}\right)=$ $h\left(s_{2}\right) \circ h\left(s_{1}\right)$ for all $s_{1}, s_{2} \in S$. We call such a mapping a homomorphism (rather than antihomomorphism) because in a product $s_{1} s_{2}$ we consider $s_{1}$ as the first factor, while in the product of maps $m_{2} \circ m_{1}$ it is natural to consider $m_{1}$ as the first factor.

A map $m$ is called constant if $m\left\langle a_{1}\right\rangle=m\left\langle a_{2}\right\rangle$ for all $a_{1}, a_{2} \in \operatorname{pr}_{1} m$. Clearly, $m$ is a constant partial transformation if and only if $\left|\mathrm{pr}_{2} m\right| \leqslant 1$, and $\left|\mathrm{pr}_{2} m\right|=0$ is possible if $m=\varnothing$, the empty map.

If $m_{1}, m_{2}$ are maps and $m_{2}$ is constant, then $m_{2} \circ m_{1}$ and $m_{1} \circ m_{2}$ are constant, i.e. the constant maps form an ideal of $\mathscr{B}_{A}$.

In this paper we characterize those semigroups which are isomorphic to semigroups of constant maps and give some properties of such semigroups. The main results of this paper were announced (without proofs or with brief outlines of proofs) in [4]. Theorem 5 of this paper was stated in [4] not in its right form. Theorem 3 of this paper (for finite semigroups) was also published in [3].

The following result is both trivial and well-known, so we omit its obvious proof.

THEOREM 1. A semigroup is isomorphic to a semigroup of constant total transformations of a set if and only if it is a right zero semigroup.

REMARK. If $h: S \rightarrow \mathscr{B}_{A}$ is defined to be a homomorphism in case $h\left(s_{1} s_{2}\right)=$ $h\left(s_{1}\right) \circ h\left(s_{2}\right)$, then right zero semigroups should be replaced by left zero ones in Theorem 1.

The triviality and simplicity of semigroups characterized in Theorem 1 may suggest that semigroups isomorphic to semigroups of constant maps (i.e. semigroups of constant maps considered "up to isomorphism," or "from an abstract standpoint") are rather trivial. As we shall see this is not so (even though semigroups of constant maps do form a very restrictive class of semigroups).

A semigroup $S$ is called a rectangular 0 -band if $S$ is a completely 0 -simple semigroup with a trivial structure group. The Suschkewitsch-Rees Representation Theorem for completely 0 -simple semigroups gives, as a by-product, the following alternative definition of rectangular 0-bands.

Let $A, B$ be nonempty sets, let $m \subset B \times A$ be a binary relation between the elements of $B$ and $A$. Suppose that $\mathrm{pr}_{1} m=B, \mathrm{pr}_{2} m=A$ (here the projections of $m$ have the obvious meaning; e.g. $\left.\operatorname{pr}_{1} m=\{b \in B:(\exists a \in A)((b, a) \in m]\}\right)$. Suppose that 0 is an element not belonging to the Cartesian product $A \times B$. Let $[\mathrm{m}]^{0}$ 
denote the set $(A \times B)^{0}=(A \times B) \cup\{0\}$ with the following binary multiplication:

(1) if $\left(b_{1}, a_{2}\right) \in m$, then $\left(a_{1}, b_{1}\right)\left(a_{2}, b_{2}\right)=\left(a_{1}, b_{2}\right)$;

(2) all other products equal 0 (in particular, $0 x=x 0=0$ for all $x \in(A \times B)^{0}$, and $\left(a_{1}, b_{1}\right)\left(a_{2}, b_{2}\right)=0$ whenever $\left.\left(b_{1}, a_{2}\right) \notin m\right)$.

Proposition 1. A semigroup $S$ is a rectangular 0 -band if and only if it is isomorphic to a semigroup of the form $[m]^{0}$.

Indeed, representing $m$ as a binary Boolean matrix $P$ (i.e. considering $B$ and $A$ as sets of rows and columns of $P$, respectively, with 1 standing at the intersection of the $b$ th row and $a$ th column if $(b, a) \in m, 0$ standing at the intersection otherwise) we arrive at a regular sandwich matrix over the trivial group 1 . Then $[m]^{0}$ is isomorphic to the Rees semigroup $\mathscr{M}^{0}[A, B, 1 ; P]$ which implies Proposition 1.

An equivalent form of the following Proposition 2 can be found in [2].

Proposition 2. A semigroup $S$ is a rectangular 0 -band if and only if it has a zero, 0 , and satisfies the following conditions:

(1) for every $x, y \in S, x y x=x$ or $x y x=0$;

(2) for every $x, y \in S, x \neq 0, y \neq 0$, there exists $z \in S$ such that $x z y \neq 0$.

Proof. Suppose $S$ is a rectangular 0-band. Without loss of generality, $S=[m]^{0}$ for suitable $m \subset B \times A$. If $x$ or $y$ is 0 , then $x y x=0$. Let $x=\left(a_{1}, b_{1}\right), y=\left(a_{2}, b_{2}\right)$. Then $x y x=x$ if $\left(b_{1}, a_{2}\right) \in m,\left(b_{2}, a_{1}\right) \in m$. Otherwise $x y x=0$. Since, by assumption, $\operatorname{pr}_{1} m=B$ and $\operatorname{pr}_{2} m=A$, there exist $a \in A$ and $b \in B$ such that $\left(b_{1}, a\right) \in m$ and $\left(b, a_{2}\right) \in m$. Let $z=(a, b)$. Then $x z y=\left(a_{1}, b_{2}\right) \neq 0$. Thus conditions (1) and (2) hold for every rectangular 0-band.

Now suppose that $S$ is a semigroup with zero satisfying conditions (1) and (2).

By (2) for every $x \in S$ there exists $z \in S$ such that $x z x=x$, i.e. $S$ is regular. Suppose $e, f$ are idempotents of $S, e=e f=f e, f \neq 0$. Then $e=f e=f e f$. By (1), $f e f$ is $f$ or 0 , i.e. $e=0$ or $e=f$. Thus, every nonzero idempotent of $S$ is primitive. If $e$ and $f$ are nonzero idempotents of $S$, then $e S f \neq 0$ by (2). Thus $S$ is completely 0 -simple (cf. Exercise 2.7.11 of [1]).

If $s, t$ belong to a subgroup of $S$, then sts belongs to the same subgroup. If $s t s=0$, then $s=t=0$. If $s t s=s$, then $s=t^{-1}$. Since this holds for any two elements of the subgroup, $s=t$. Thus, all subgroups of $S$ are trivial. By Proposition 1 and the Suschkewitsch-Rees Theorem $S$ is a rectangular 0-band.

A hypergraph is any triple $(A, B, h)$, where $A$ and $B$ are nonempty sets, a set of vertices and a set of edges, respectively, while $h \subset A \times B$ is a binary relation 
called the incidence relation. If $(a, b) \in h$, the vertex $a$ is called incident to the edge $b$. This definition corresponds to definitions of hypergraphs one can find in various sources.

Two hypergraphs $\left(A_{1}, B_{1}, h_{1}\right)$ and $\left(A_{2}, B_{2}, h_{2}\right)$ are called isomorphic if there exist a bijection $\alpha$ of $A_{1}$ onto $A_{2}$ and a bijection $\beta$ of $B_{1}$ onto $B_{2}$ such that $\left(a_{1}, b_{1}\right) \in h_{1} \Leftrightarrow\left(\alpha\left(a_{1}\right), \beta\left(b_{1}\right)\right) \in h_{2}$ for all $a_{1} \in A_{1}, b_{1} \in B_{1}$.

A vertex (edge) which is not incident to any edge (vertex) is called isolated.

Suppose $(A, B, h)$ is a hypergraph without isolated vertices and edges. Then $[h]^{0}$ is a rectangular 0 -band with the set $(B \times A) \cup\{0\}$ of elements.

Proposition 3. Two hypergraphs $\left(A_{1}, B_{1}, h_{1}\right)$ and $\left(A_{2}, B_{2}, h_{2}\right)$ without isolated vertices and edges are isomorphic if and only if the rectangular 0 -bands $\left[h_{1}\right]^{0}$ and $\left[h_{2}\right]^{0}$ are isomorphic.

Proof. The "only if" part is trivial. To prove the "if" part, suppose $f$ is an isomorphism of $\left[h_{1}\right]$ onto $\left[h_{2}\right]$. Then $f(0)=0$ and $f\left(B_{1} \times A_{1}\right)=B_{2} \times A_{2}$. Two elements $\left(b_{1}, a_{1}\right),\left(b_{2}, a_{2}\right) \in\left[h_{1}\right]^{0}$ are $\mathscr{R}$-related ( $\mathscr{L}$-related) if and only if $b_{1}=b_{2}$ $\left(a_{1}=a_{2}\right)$. Since $f$, being an isomorphism, preserves the Green relations, $f\left(b_{1}, a_{1}\right)$ and $f\left(b_{2}, a_{2}\right)$ are $\mathscr{R}$-related ( $\mathscr{L}$-related) in $\left[h_{2}\right]^{0}$ if and only if $\left(b_{1}, a_{1}\right)$ and $\left(b_{2}, a_{2}\right)$ are $\mathscr{R}$-related ( $\mathscr{L}$-related) in $\left[h_{1}\right]^{0}$. Thus, there exist bijections $f_{1}: B_{1} \rightarrow B_{2}$ and $f_{2}: A_{1} \rightarrow A_{2}$ such that $f(b, a)=\left(f_{1}(b), f_{2}(a)\right)$ for all $(b, a) \in\left[h_{1}\right]^{0}$. Since $\left(b_{1}, a_{1}\right)\left(b_{2}, a_{2}\right) \neq 0$ if and only if $\left(a_{1}, b_{2}\right) \in h_{1}$, it follows easily that $\left(f_{2}, f_{1}\right)$ is an isomorphism of $\left(A_{1}, B_{1}, h_{1}\right)$ onto $\left(A_{2}, B_{2}, h_{2}\right)$.

In fact, the proof of Proposition 3 implies a stronger result: the categories of hypergraphs without isolated vertices and edges and of rectangular 0-semigroups are equivalent (the morphisms in each of the categories are isomorphisms). A generalization of Proposition 3 is proved in [6].

Thus, completely 0 -simple semigroups with trivial structure groups characterize hypergraphs without isolated vertices and edges up to isomorphism.

A semigroup $S$ is called rectangular 0 -subband if $S$ is isomorphically embeddable in a rectangular 0 -band.

THEOREM 2. The following conditions are equivalent for every semigroup $S$ :

(1) $S$ is isomorphic to a semigroup of constant partial transformations;

(2) $S$ is isomorphic to a semigroup of constant maps;

(3) $S$ is a rectangular $0-$ subband.

Proof. Every partial transformation is a map, so the implication (1) $\Rightarrow(2)$ is trivial.

(2) $\Rightarrow$ (3). Suppose (2) holds. It is easy to see that the semigroup of all constant maps of any set $A$ is a rectangular 0 -band. Since $S$ is isomorphically embeddable in a semigroup of all constant maps, $S$ must be a rectangular 0 -subband. 
(3) $\Rightarrow$ (1). Suppose $S$ is isomorphic to a subsemigroup of a rectangular 0-band $T$. If $T$ is isomorphic to a semigroup of constant partial transformations, then $S$ is isomorphic to such a semigroup. Thus, without loss of generality, we may suppose that $S$ is a rectangular 0-band. Suppose $S=[m]^{0}$, where $m \subset B \times A, \operatorname{pr}_{1} m=B$, $\operatorname{pr}_{2} m=A, A \cap B=\varnothing$. For every $(a, b) \in S$ define $f(a, b)=\left(m^{-1}\langle a\rangle \cup\{a\}\right)$ $\times\{b\}$, and define $f(0)=\varnothing$. Clearly, for every $x \in S, f(x)$ is a constant partial transformation of the set $A \cup B$. It is obvious that $f(a, b) \neq \varnothing$ and that $f(x)=f(y)$ implies $x=y$. Indeed, if $x$ or $y$ is 0 , then $f(x)=f(y)=\varnothing$, so $x=y=0$. If $x=\left(a_{1}, b_{1}\right), y=\left(a_{2}, b_{2}\right)$, then $\left\{b_{1}\right\}=\operatorname{pr}_{2} f(x)=\operatorname{pr}_{2} f(y)=\left\{b_{2}\right\}$, and hence $b_{1}=b_{2}$. Also $\left\{a_{1}\right\}=A \cap \operatorname{pr}_{1} f(x)=A \cap \operatorname{pr}_{1} f(y)=\left\{a_{2}\right\}$, and hence $a_{1}=a_{2}$, so $x=y$. Thus $f$ is one-to-one. If $x$ or $y$ is 0 , then $f(x y)=f(y) \circ f(x)=$ $\varnothing$. Let $x=\left(a_{1}, b_{1}\right), y=\left(a_{2}, b_{2}\right)$. Then $x y \neq 0$ if and only if $\left(b_{1}, a_{2}\right) \in m$, i.e. if $b_{1} \in m^{-1}\left\langle a_{2}\right\rangle$. On the other hand, $f(y) \circ f(x) \neq \varnothing$ if and only if $b_{1} \in \operatorname{pr}_{1} f(y)$, i.e. $b_{1} \in m^{-1}\left\langle a_{2}\right\rangle$. Hence $f(x y)=f(y) \circ f(x)$ if $x y=0$. Now suppose that $x y \neq 0$. Then $x y=\left(a_{1}, b_{2}\right)$ and $f(x y)=\left(m^{-1}\left\langle a_{1}\right\rangle \cup\left\{a_{1}\right\}\right) \times\left\{b_{2}\right\}=f(y) \circ f(x)$. Thus, $f$ is an isomorphism of $S$ onto a semigroup of constant partial transformations. This completes our proof.

It follows from the Fundamental Theorem on Relation Algebras (see [5]) that the class of all rectangular 0 -subbands is a class of abstract relation algebras, and so it can be characterized by a system of elementary axioms. The same conclusion follows from the observation that all rectangular 0-subbands are a class of all subalgebras of an axiomatizable class of algebras (rectangular 0-bands) and application of a result of Tarski [7]. In what follows, we present a system of elementary axioms for rectangular 0 -subbands.

Suppose $S$ is a rectangular 0 -subband and $S$ has no zero. It follows from Proposition 2 that, for every $x, y \in S, x y x=x$. Thus $S$ is a rectangular band. Conversely, if $S$ is a rectangular band, then $S^{0}$ is obviously a rectangular 0 -band, hence $S$ is a rectangular 0 -subband.

Thus, rectangular 0 -subbands without zero are precisely rectangular bands.

In what follows, we assume that rectangular 0 -subbands to be considered always contain zero. While inessential, this assumption simplifies our argument.

We adopt the following notation: if $H$ is a subset of a semigroup $S$ with zero, then $H^{-}$denotes $H \backslash\{0\}$. In particular, $H^{-}=H$ whenever $0 \notin H$. If $s \in S$, then $(s)$, and $(s)$, denote the principal left and right ideals generated by $s$. We introduce the following binary relations $\sigma_{1}$ and $\sigma_{2}$ on $S$ :

$$
\sigma_{1}=\left\{(s, t) \in S \times S:\left(s_{1}\right)_{r}^{-}\left(s_{2}\right)_{r}^{-}\right\}, \quad \sigma_{2}=\left\{(s, t) \in S \times S:\left(s_{1}\right)_{i}^{-}\left(s_{2}\right)_{l}^{-}\right\} \text {, }
$$

where $A B$ means $A \cap B \neq \varnothing$ for any two sets $A$ and $B$. Clearly, both $\sigma_{1}$ and $\sigma_{2}$ are symmetric binary relations.

Let $\Delta_{S}$ be the diagonal of $S \times S$, i.e. $\Delta_{S}=\{(s, t) \in S \times S: s=t\}$. Let $\pi_{i}$ denote the transitive closure of $\sigma_{i} \cup \Delta_{S}, i=1,2$. Then $\pi_{i}$ is transitive by definition, it is reflexive and symmetric because $\sigma_{i} \cup \Delta_{S}$ is. In other words, $\pi_{i}$ is an 
equivalence relation on $S$. Let $\pi=\pi_{1} \cap \pi_{2}$. Then $\pi$ is an equivalence relation on $S$. If $S$ is a semigroup with zero 0 satisfying the condition $x y z=0 \Rightarrow(x y=0$ or $y z=0$ ) for all $x, y, z \in S$, then $S$ is called categorial at 0.

A semigroup $S$ is called quasiprimitive if $\pi=\Delta_{S}$.

THEOREM 3. A semigroup $S$ is a rectangular 0 -subband if and only if $S$ is a rectangular band or $S$ is quasiprimitive and categorial at 0 .

Proof. As explained above, we may assume that $S$ has zero 0 .

First suppose that $S$ is a rectangular 0-subband, say, $S \subset[m]^{0}$ for some $m \subset B \times A$. It is easy to see that $0 \in S$. Suppose that $x, y \in S$ and $(x, y) \in \sigma_{1}$. This means $(x)_{r}^{-}(y)_{r}^{-}$, i.e. there exists $u, v \in S^{1}$ such that $x u=y v \neq 0$. Now, it follows that none of $x, y, u, v$ is 0 . Suppose $x=\left(a_{1}, b_{1}\right), y=(a, b)$. Then $x u=u v \neq 0$ implies $a_{1}=a$. Now suppose $(x, y) \in \pi_{1}$. If $x \neq y$, then there exist $s_{1}, s_{2}, \ldots, s_{n} \in S$ such that $x=s_{1}, y=s_{n}$, and $\left(s_{i}\right)_{r}^{-}\left(s_{i+1}\right)_{r}^{-}$for $i=1, \ldots, n-1$. It follows that $s_{i} \neq 0$. Let $s_{i}=\left(a_{i}, b_{i}\right)$. Then, as we have just seen, $a_{i}=a_{i+1}$ for all $i$. In particular, $a_{1}=a_{2}=\cdots=a_{n}=a$. Analogously, if $(x, y) \in \pi_{2}$ and $x \neq y$, then $b_{1}=b$. Thus, if $(x, y) \in \pi$ and $x \neq y$, then $a_{1}=a, b_{1}=b$, i.e. $x=y$ which contradicts our assumption. Thus $\pi=\Delta_{S}$, i.e. $S$ is quasiprimitive. Next, suppose that $x y z=0$ for $x, y, z \in S$. If $x, y$, or $z$ is 0 , then $x y=0$ or $y z=0$. If $x=\left(a_{1}, b_{1}\right), y=\left(a_{2}, b_{2}\right), z=\left(a_{3}, b_{3}\right)$ then $\left(b_{1}, a_{2}\right) \notin m$ or $\left(b_{2}, a_{3}\right) \notin m$ (otherwise $\left.x y z=\left(a_{1}, b_{3}\right) \neq 0\right)$. In the former case $x y=0$, in the latter case $y z=0$. Thus $S$ is categorial at 0 .

Conversely, suppose that $S$ is a quasiprimitive semigroup which is categorial at 0 . We will prove that $S$ is isomorphic to a semigroup of constant maps.

For each $s \in S$ we define a map $f(s)$ of the set $S^{1} \times S^{1}$ into itself.

Let $\nu$ be a binary relation on $S^{1}$ defined as follows:

$$
\nu=\left\{(s, t) \in S^{1} \times S^{1}: s t \neq 0\right\} .
$$

Define

$$
f(s)=\left(\pi_{1}\langle s\rangle \times \nu^{-1}\langle s\rangle\right) \times\left(\nu\langle s\rangle \times \pi_{2}\langle s\rangle\right) .
$$

If $s=0$, then $\nu\langle s\rangle=\varnothing$, and hence $f(0)=\varnothing$. If $s \neq 0$, then $s \in \pi_{1}\langle s\rangle, 1 \in \nu\langle s\rangle$, $1 \in \nu^{-1}\langle s\rangle$, and $s \in \pi_{2}\langle s\rangle$. Thus $((s, 1),(1, s)) \in f(s)$ and $f(s) \neq \varnothing$. Suppose $f\left(s_{1}\right)=f\left(s_{2}\right)$ for $s_{1}, s_{2} \in S$. If one of the elements $s_{1}, s_{2}$ is 0 , then $f\left(s_{1}\right)=f\left(s_{2}\right)=$ $\varnothing$, and hence $s_{1}=s_{2}=0$. Suppose that neither of the elements is 0 . Then $f\left(s_{1}\right) \neq \varnothing \neq f\left(s_{2}\right)$. Hence $\pi_{1}\left\langle s_{1}\right\rangle=\pi_{1}\left\langle s_{2}\right\rangle$ and $\pi_{2}\left\langle s_{1}\right\rangle=\pi_{2}\left\langle s_{2}\right\rangle$. It follows that $\pi\left\langle s_{1}\right\rangle=\pi\left\langle s_{2}\right\rangle$. Since $\pi$ is an equivalence relation, this means that $\left(s_{1}, s_{2}\right) \in \pi$. However, $S$ is quasiprimitive. Thus $s_{1}=s_{2}$ which shows that $f$ is one-to-one. It is clear that $f(s)$ is a rectangular binary relation (= a map) for every $s \in S$. It remains to prove that

$$
f(x y)=f(y) \circ f(x) \quad \text { for each } x, y \in S .
$$


If $x$ or $y$ is 0 then both sides of (1) are $\varnothing$, and (1) holds. Suppose now that $x \neq 0, y \neq 0$. First we prove that if one part of (1) is $\varnothing$, then (1) holds.

Clearly, $f(x y) \neq \varnothing$ if and only if $x y \neq 0$, i.e. $(x, y) \in \nu$ or, equivalently, $y \in \nu\langle x\rangle$ and $x \in \nu^{-1}\langle y\rangle$. Now, $f(y) \circ f(x) \neq \varnothing$ precisely when $\operatorname{pr}_{2} f(x)$ $\operatorname{pr}_{1} f(y)$, i.e. when

$$
\nu\langle x\rangle \pi_{1}\langle y\rangle, \pi_{2}\langle x\rangle \nu^{-1}\langle y\rangle .
$$

If $x y \neq 0$ then $y \in \nu\langle x\rangle$. Since $\pi_{1}$ is reflexive, $y \in \pi_{1}\langle y\rangle$. Analogously, $x$ belongs to both $\pi_{2}\langle x\rangle$ and $\nu^{-1}\langle y\rangle$. Thus (2) holds. In other words, $f(x y) \neq \varnothing \Rightarrow$ $f(y) \circ f(x) \neq \varnothing$. The converse implication will follow from Lemma 1 .

Lemma 1. If $(u, v) \in \pi_{1}$ and $x u \neq 0$, then $(x u, x v) \in \pi_{1}$ and $x v \neq 0$. Analogously, if $(u, v) \in \pi_{2}$ and $u x \neq 0$, then $(u x, v x) \in \pi_{2}$ and $v x \neq 0$.

Proof. Suppose that $(u, v) \in \pi_{1}$ and $x u \neq 0$. By the definition of $\pi_{1},(u, v) \in \pi_{1}$ means that there exist $n$ and $s_{1}, s_{2}, \ldots, s_{n} \in S$ such that $u=s_{1}, s_{n}=v$ and $\left(s_{i}, s_{i+1}\right) \in \sigma_{1} \cup \Delta_{S}$ for all $i=1, \ldots, n-1$. Now, if $\left(s_{i}, s_{i+1}\right) \in \Delta_{S}$ for some $i$, then $s_{i}=s_{i+1}$ and we can just make the sequence $s_{1}, \ldots, s_{n}$ shorter by skipping $s_{i+1}$ and considering the sequence $s_{1}, \ldots, s_{i}, s_{i+2}, \ldots, s_{n}$ instead. Thus we may suppose that $\left(s_{i}, s_{i+1}\right) \in \sigma_{1}$ for all $i=1, \ldots, n-1$. This means that $\left(s_{i}\right)_{r}^{-} \ell\left(s_{i=1}\right)_{r}^{-}$, i.e., $s_{i} x_{i}=s_{i+1} y_{i+1} \neq 0$ for some $x_{i}, y_{i+1} \in S^{1}$. Multiplying each of these equalities by $x$ on the left, we get $\left(x s_{i}\right) x_{i}=\left(x s_{i+1}\right) y_{i+1}$. Now, $x s_{1}=x u \neq 0$ by our condition. Also, $s_{1} x_{1} \neq 0$ because $s_{i} x_{i} \neq 0$ for all $i$. Since $S$ is categorial at 0 , we obtain that $0 \neq x\left(s_{1} x_{1}\right)=x\left(s_{2} y_{2}\right)=\left(x s_{2}\right) y_{2}$, hence $x s_{2} \neq 0$. Thus, $x s_{2} \neq 0$ and $s_{2} x_{2} \neq 0$. Since $S$ is categorial at $0,\left(x s_{2}\right) x_{2} \neq 0$. Therefore $\left(x s_{3}\right) y_{3} \neq 0$ which implies $x s_{3} \neq 0$. Going on, we obtain $x s_{i} \neq 0$ for all $i$. Finally $x s_{i} \neq 0$ and $s_{i} x_{i} \neq 0$ imply $\left(x s_{i}\right) x_{i} \neq 0$. Then $\left(x s_{i}\right) x_{i}=\left(x s_{i+1}\right) y_{i+1} \neq 0$ for all $i$. This implies $\left(x s_{i}, x s_{i+1}\right) \in \sigma_{1}$ for all $i$, and therefore $\left(x s_{1}, x s_{n}\right) \in \pi_{1}$. In other words, $(x u, x v)$ $\in \pi_{1}$. Also, $x v=x s_{n} \neq 0$. The second part of the lemma may be proved analogously.

Now we get back to our proof of Theorem 3. Suppose that $f(y) \circ f(x) \neq \varnothing$. As we have seen, this means that relations (2) hold. Thus, there exist $u, v \in S$ such that $u \in \nu\langle x\rangle \cap \pi_{1}\langle y\rangle$ and $v \in \pi_{2}\langle x\rangle \cap \nu^{-1}\langle y\rangle$. In other words, $x u \neq 0$ and $(y, u) \in \pi_{1} ;$ also $(x, v) \in \pi_{2}$ and $v y \neq 0$. By Lemma $1(x y, x u) \in \pi_{1}$ and $(x y, v y)$ $\in \pi_{2}$ and $x y \neq 0$. Thus, $f(x y) \neq \varnothing$.

Hence $f(x y) \neq \varnothing \Leftrightarrow f(y) \circ f(x) \neq \varnothing$. Suppose one side of the above equivalence does not hold. Then both sides of (1) equal $\varnothing$, i.e. (1) holds.

It remains to consider the case when $f(x y) \neq \varnothing$ and $f(y) \circ f(x) \neq \varnothing$. Suppose $s \in \nu^{-1}\langle x\rangle$ for some $s \in S^{1}$. This means $s x \neq 0$. Since $x y \neq 0$ and $S$ is categorial at 0 , we get $s x y \neq 0$, whence $s \in \nu^{-1}\langle x y\rangle$. Conversely, if $s \in \nu^{-1}\langle x y\rangle$, then $s x y \neq 0, s x \neq 0$, and $s \in \nu^{-1}\langle x\rangle$. Thus $\nu^{-1}\langle x\rangle=\nu^{-1}\langle x y\rangle$. Analogously we may prove that $\nu\langle y\rangle=\nu\langle x y\rangle$. Now, $x y \neq 0$ and $x y \in(x)_{r}^{-} \cap(x y)_{r}^{-}$, and hence 
$(x, x y) \in \sigma_{1} \subset \pi_{1}$. Thus $\pi_{1}\langle x\rangle=\pi_{1}\langle x y\rangle$. Analogously we may prove that $\pi_{2}\langle y\rangle$ $=\pi_{2}\langle x y\rangle$. Since $f(y) \circ f(x) \neq \varnothing$, we have $f(y) \circ f(x)=\left(\pi_{1}\langle x\rangle \times \nu^{-1}\langle x\rangle\right) \times$ $\left(\nu\langle y\rangle \times \pi_{2}\langle y\rangle\right)=\left(\pi_{1}\langle x y\rangle \times \nu^{-1}\langle x y\rangle\right) \times\left(\nu\langle x y\rangle \times \pi_{2}\langle x y\rangle\right)=f(x y)$. Thus (1) always holds. We have proved that $f$ is an isomorphism of $S$ onto a semigroup of constant maps. By Theorem 2, $S$ is a rectangular 0 -subband. This completes the proof of Theorem 3.

Our next objective is to characterize the class of rectangular 0 -subbands by a system of elementary axioms.

THEOREM 4. A semigroup $S$ is a rectangular 0 -subband if and only if it is a rectangular band (i.e. satisfies the identity $x y x=x$ ) or $S$ contains a zero and satisfies the following axioms:

$$
x y z=0 \Rightarrow x y=0 \vee y z=0,
$$

and, for every $n \geqslant 1$,

$$
\begin{aligned}
& s_{0} x_{0}=s_{1} y_{1} \neq 0 \wedge u_{0} t_{0}=v_{1} t_{1} \neq 0 \wedge \\
& s_{1} x_{1}=s_{2} y_{2} \neq 0 \wedge u_{1} t_{1}=v_{2} t_{2} \neq 0 \wedge \\
& \text {....... } \\
& s_{n-1} x_{n-1}=s_{n} y_{n} \neq 0 \wedge u_{n-1} t_{n-1}=v_{n} t_{n} \neq 0 \wedge \\
& s_{0}=t_{0} \wedge s_{n}=t_{n} \Rightarrow s_{0}=s_{n},
\end{aligned}
$$

where $\vee$ and $\wedge$ are the disjunction and conjunction connectives respectively, the variables $x, y, z, s_{0}, \ldots, s_{n}, t_{0}, \ldots, t_{n}$ take arbitrary values in $S$, while the variables $x_{0}, \ldots, x_{n-1}, y_{1}, \ldots, y_{n}, u_{0}, \ldots, u_{n-1}, v_{1}, \ldots, v_{n}$ take arbitrary values in $S^{1}$.

Proof. First we express the axioms $C$ and $Q_{n}$ in an equivalent form. Of course, $C$ means just that $S$ is categorial at 0 . Now, $s_{i} x_{i}=s_{i+1} y_{i+1} \neq 0$ means that $\left(s_{i}, s_{i+1}\right) \in \sigma_{1}$, and $u_{i} t_{i}=v_{i+1} t_{i+1} \neq 0$ means that $\left(t_{i}, t_{i+1}\right) \in \sigma_{2}$. Thus it follows from the antecedent of $Q_{n}$ that $\left(s_{0}, s_{n}\right) \in \pi_{1},\left(t_{0}, t_{n}\right) \in \pi_{2}, s_{0}=t_{0}, s_{n}=t_{n}$. We conclude that $\left(s_{0}, s_{n}\right) \in \pi_{1} \cap \pi_{2}=\pi$. Now, if $S$ is quasiprimitive, then $s_{0}=s_{n}$ and $Q_{n}$ holds. Therefore, if $S$ is a rectangular 0 -subband then, by Theorem $3, S$ is categorial at 0 and quasiprimitive, and $C$ and $Q_{n}$ hold.

Now suppose that $S$ is a semigroup with 0 in which $C$ and all $Q_{n}$ hold. Because of $C, S$ is categorial at 0 . To prove that $S$ is quasiprimitive, suppose that $(x, y) \in \pi$ for some $x, y \in S$. We must prove that $x=y$. Now, $(0, s) \in \sigma_{1} \cup \Delta_{S}$ implies $0=s$. Indeed, $(0, s) \in \sigma_{1}$ means that $(0)_{r}^{-} \chi(s)_{r}^{-}$. Since $(0)_{r}^{-}=\varnothing,(0, s)$ $\in \sigma_{1}$ is impossible. Thus $(0, s) \in \Delta_{s}$ and $0=s$. It follows that $(0, s) \in \pi_{1}$ implies $0=s$. Therefore, if $x$ or $y$ is 0 , then $x=y=0$. Suppose that $x \neq 0, y \neq 0$. Then $(x, y) \in \pi_{1}$ and $(x, y) \in \pi_{2}$. Now, $(x, y) \in \pi_{1}$ means that there exist $s_{0}, \ldots, s_{n} \in S$ such that $x=s_{0}, s_{n}=y$, and $\left(s_{i}, s_{i+1}\right) \in \sigma_{1} \cup \Delta_{S}$ for every $i=0, \ldots, n-1$. Analogously, $(x, y) \in \pi_{2}$ means that there exist $t_{0}, \ldots, t_{m} \in S$ such that $x=t_{0}$, $t_{m}=y$, and $\left(t_{i}, t_{i+1}\right) \in \sigma_{l} \cup \Delta_{S}$ for every $i=0, \ldots, m-1$. Here all the elements 
$s_{i}, t_{i}$ are different from 0 . If $\left(s_{i}, s_{i+1}\right) \in \Delta_{s}$, then $s_{i}=s_{i+1}$ and $s_{i} \in\left(s_{i}\right)_{r}^{-}=\left(s_{i+1}\right)_{r}^{-}$, whence $\left(s_{i}, s_{i+1}\right) \in \sigma_{1}$. Thus, without loss of generality, we may suppose that $\left(s_{i}, s_{i+1}\right) \in \sigma_{1}$ for $i=0, \ldots, n-1$, and $\left(t_{i}, t_{i+1}\right) \in \sigma_{2}$ for $i=0, \ldots, m-1$. Also, we may suppose that $m=n$. Indeed, if $m<n$, add $t_{m+1}, \ldots, t_{n}$ all equal to $t_{m}$; if $n<m$, add $s_{n+1}, \ldots, s_{m}$ all equal to $s_{n}$.

Thus, without loss of generality, we may suppose that there exist $s_{0}, \ldots, s_{n}$, $t_{0}, \ldots, t_{n} \in S$ such that $x=s_{0}=t_{0}, y=s_{n}=t_{n}$, and $\left(s_{i}, s_{i+1}\right) \in \sigma_{1},\left(t_{i}, t_{i+1}\right) \in \sigma_{2}$ for all $i=0, \ldots, n-1$. Now, $\left(s_{i}, s_{i+1}\right) \in \sigma_{1}$ means $\left(s_{i}\right)_{r}^{-} \ell\left(s_{i+1}\right)_{r}^{-}$, i.e. $s_{i} x_{i}=$ $s_{i+1} y_{i+1} \neq 0$, for certain $x_{i}, y_{i+1} \in S^{1}$, while $\left(t_{i}, t_{i+1}\right) \in \sigma_{2}$ means $\left(t_{i}\right)_{i} \backslash\left(t_{i+1}\right)_{i}^{-}$, i.e. $u_{i} t_{i}=v_{i+1} t_{i+1} \neq 0$ for certain $u_{i}, v_{i+1} \in S^{1}$. Thus the antecedent of $Q_{n}$ holds for our elements $s_{i}, t_{i}, x_{i}, y_{i}, u_{i}, v_{i}$. By $Q_{n}, s_{0}=s_{n}$, i.e. $x=y$ and $S$ is quasiprimitive. By Theorem $3, S$ is a rectangular 0 -subband. This completes the proof of Theorem 4.

Remarks. (1) Syntactically, the axioms $C$ and $Q_{n}$ are not formulas of the first-order predicate calculus for the theory of semigroups because an extralogical symbol 0 is used in them. Of course, one may consider 0 as a logical symbol for a nullary operator, and instead of semigroups, consider semigroups with 0 . However, it is obvious that both $C$ and $Q_{n}$ are equivalent to formulas in the language of the theory of semigroups. For example, $C$ is obviously equivalent to $x y \neq 0 \wedge$ $y z \neq 0 \Rightarrow x y z \neq 0$.

Now, any formula $w \neq 0$, where $w$ is a term ( $=$ a word) in the semigroup language, is obviously equivalent to the formula $(\exists u)[w u \neq w \vee u w \neq w]$, which belongs to the semigroup language.

(2) We have characterized rectangular 0 -subbands with and without 0 using different systems of axioms. However, one may consider $C$ and $Q_{n}$ as axioms for all rectangular 0 -subbands. If a semigroup $S$ has no zero then $(\forall x)[x \neq 0]$ is obviously true in $S$ (or, if one prefers the semigroup language without 0 symbol, $(\forall x)(\exists y)[x y \neq x \vee y x \neq x]$ is always true). However, for semigroups without 0 , the infinite system of axioms produced by Theorem 4 can be substantially simplified, while for semigroups with 0 this is not the case.

It can be proved that the system of axioms given in Theorem 4 is not equivalent to any finite system of elementary axioms. We omit the proof because it is too tedious. In fact, for each $n \geqslant 2$ we can construct a semigroup $S_{n}$ which satisfies $C$ and $Q_{i}$ for $i<n$ but which does not satisfy $Q_{n}$. The semigroup $S_{n}$ is finitely presented, and the proof that $Q_{i}, i<n$, hold in $S_{n}$ consists of a long and detailed checking of various conditions which may be satisfied by words representing elements of $S_{n}$. Thus, $Q_{n}$ does not follow from $C, Q_{1}, \ldots, Q_{n-1}$, which shows that the infinite system of axioms $\left\{C, Q_{1}, Q_{2}, \ldots\right\}$ is not equivalent to any of its finite subsystems. By the Completeness Theorem for the first order predicate calculus it 
follows that our system of axioms is not equivalent to any finite system of elementary axioms.

An involuted semigroup is an algebra $\left(S ; \cdot,{ }^{-1}\right)$, where $\cdot$ is an associative binary operation, ${ }^{-1}$ is a unary operation on the set $S$, and the following identities hold: $\left(x^{-1}\right)^{-1}=x,(x y)^{-1}=y^{-1} x^{-1}$. If $\Phi$ is a nonempty set of maps which is closed both under composition $\circ$ and conversion ${ }^{-1}$ of maps, then $\left(\Phi ; \circ,{ }^{-1}\right)$ is an involuted semigroup of maps.

The following result gives an abstract characterization of involuted semigroups which are isomorphic to involuted semigroups of constant maps:

THEOREM 5. An involuted semigroup $S$ is isomorphic to an involuted semigroup of constant maps if and only if $S$ satisfies the following conditions:

(1) $x x^{-1} x=x$;

(2) $x x^{-1}=y y^{-1}$ and $x^{-1} x=y^{-1} y \Rightarrow x=y$;

(3) $x y \neq 0 \Rightarrow x x^{-1}=x y y^{-1} x^{-1}$,

where $x y \neq 0$ means that $x y$ is not a zero of the semigroup $S$ (we do not assume that $S$ contains a zero).

Proof. Suppose that $S$ is isomorphic to an involuted semigroup of constant maps. Without loss of generality, we may assume that $S$ is an involuted semigroup of constant maps of a set $A$. It is clear that $x \circ x^{-1} \circ x=x$ for every $x \in S$. Thus, (1) holds. Suppose that $x, y \in S$ and $x^{-1} \circ x=y^{-1} \circ y, x \circ x^{-1}=y \circ y^{-1}$. Since $\operatorname{pr}_{1}\left(x^{-1} \circ x\right)=\operatorname{pr}_{1} x$ and $\operatorname{pr}_{2}\left(x \circ x^{-1}\right)=\operatorname{pr}_{2} x$, it follows that $\operatorname{pr}_{1} x=\operatorname{pr}_{1} y$ and $\operatorname{pr}_{2} x=\operatorname{pr}_{2} y$. Since $x$ and $y$ are constant maps, it follows that $x=y$. Indeed, for every $a \in \mathrm{pr}_{1} x, x\langle a\rangle=\mathrm{pr}_{2} x=\mathrm{pr}_{2} y=y\langle a\rangle$. Thus, condition (2) holds.

To prove that (3) holds, assume that $y \circ x \neq 0$ for some $x, y \in S$. Then $y \circ x \neq \varnothing$. It follows that $x^{-1} \circ y^{-1} \circ y \circ x \neq \varnothing$. Since $x, y, x^{-1}, y^{-1}$ are constant maps, $x^{-1} \circ y^{-1} \circ y \circ x=x^{-1} \circ x$, because $x^{-1} \circ y^{-1} \circ y \circ x=(y \circ x)^{-1} \circ(y \circ x)=$ $\operatorname{pr}_{1}(y \circ x) \times \operatorname{pr}_{2}(y \circ x)^{-1}=\operatorname{pr}_{1}(y \circ x) \times \operatorname{pr}_{1}(y \circ x)=\operatorname{pr}_{1} x \times \operatorname{pr}_{1} x=\operatorname{pr}_{1} x \times$ $\operatorname{pr}_{2} x^{-1}=x^{-1} \circ x$.

To prove sufficiency of conditions (1)-(3) suppose that they hold for an involuted semigroup $S$. Introduce two binary relations, $\sigma$ and $\mu$, on $S^{-}$:

$$
\sigma=\left\{(s, t) \in S^{-} \times S^{-}: s s^{-1}=t t^{-1}\right\} ; \quad \mu=\left\{(s, t) \in S^{-} \times S^{-}: s^{-1} t \neq 0\right\} .
$$

Clearly, $\sigma$ is an equivalence relation, while $\mu$ is reflexive and symmetric. Indeed, if $s \in S^{-}$, then $0 \neq s=s s^{-1} s$, hence $s^{-1} s \neq 0$ and $(s, s) \in \mu$. Also if $(s, t) \in \mu$, then $s^{-1} t \neq 0$, hence $t^{-1} s=\left(s^{-1} t\right)^{-1} \neq 0$ and $(t, s) \in \mu$.

A tolerance space is a pair $(A ; \rho)$, where $\rho$ is a tolerance relation (i.e. a reflexive and symmetric binary relation) on a set $A$. Two tolerance spaces $(A ; \rho)$ and $(B ; \tau)$ are called isomorphic if there exists a bijection $f: A \rightarrow B$ such that $\left(a_{1}, a_{2}\right) \in \rho \Leftrightarrow\left(f\left(a_{1}\right), f\left(a_{2}\right) \in \tau\right.$ for all $a_{1}, a_{2} \in A$. 
An intersection space is a tolerance space of the form $(A ; \rho)$, where $A$ is a set of nonempty subsets of some underlying set, while $\left(a_{1}, a_{2}\right) \in \rho \Leftrightarrow a_{1} a_{2}$, i.e. subsets $a_{1}$ and $a_{2}$ are $\rho$-related if and only if they are not disjoint.

LEMMA 2. Every tolerance space is isomorphic to an intersection space.

Proof. Let $(A ; \rho)$ be a tolerance space. For each $a \in A$ define a subset $f(a)$ of the set $A \times A: f(a)=(\{a\} \times \rho\langle a\rangle) \cup(\rho\langle a\rangle \times\{a\})$. Since $\rho$ is reflexive, $a \in$ $\rho\langle a\rangle$ and $(a, a) \in f(a)$. Thus, $f(a)$ is never empty.

Consider the intersection space on the set $\{f(a): a \in A\}$ of subsets of $A$. It follows that $\left(a_{1}, a_{2}\right) \in \rho \Leftrightarrow f\left(a_{1}\right) \backslash f\left(a_{2}\right)$. Indeed, if $\left(a_{1}, a_{2}\right) \in \rho$, then $a_{2} \in \rho\left\langle a_{1}\right\rangle$ and $\left(a_{2}, a_{1}\right) \in \rho$, hence $a \in \rho\left\langle a_{2}\right\rangle$, and therefore $\left(a_{1}, a_{2}\right) \in f\left(a_{1}\right) \cap f\left(a_{2}\right)$ and $f\left(a_{1}\right) \ell f\left(a_{2}\right)$. Conversely, if $f\left(a_{1}\right) \backslash f\left(a_{2}\right)$, then $\left(a_{1}, a_{2}\right) \in \rho$. Also, $f$ is one-to-one: for suppose that $f\left(a_{1}\right)=f\left(a_{2}\right)$. Since $\left(a_{1}, a_{1}\right) \in f\left(a_{1}\right)$, we obtain that $\left(a_{1}, a_{1}\right) \in$ $f\left(a_{2}\right)$, whence $a_{1}=a_{2}$. Thus, $f$ is the isomorphism of $(A, \rho)$ onto the intersection space we have constructed. Lemma 2 is proved.

LeMma 3. If $\left(s_{1}, s_{2}\right) \in \sigma$ and $\left(t_{1}, t_{2}\right) \in \sigma$, then $\left(s_{1}, t_{1}\right) \in \mu \Leftrightarrow\left(s_{2}, t_{2}\right) \in \mu$.

Proof. Suppose that $\left(s_{1}, s_{2}\right) \in \sigma$ and $\left(t_{1}, t_{2}\right) \in \sigma$, i.e. $s_{1} s_{1}^{-1}=s_{2} s_{2}^{-1}$ and $t_{1} t_{1}^{-1}$ $=t_{2} t_{2}^{-1}$. If $\left(s_{1}, t_{1}\right) \in \mu$, then $s_{1}^{-1} t_{1} \neq 0$, hence $0 \neq s_{1}^{-1} t_{1}=\left(s_{1}^{-1} t_{1}\right)\left(s_{1}^{-1} t_{1}\right)^{-1}\left(s_{1}^{-1} t_{1}\right)$ $=s_{1}^{-1} t_{1} t_{1}^{-1} s_{1} s_{1}^{-1} t_{2}=s_{1}^{-1} t_{2} t_{2}^{-1} s_{2} s_{2}^{-1} t_{1}$. It follows that $t_{2}^{-1} s_{2} \neq 0$, i.e. $\left(t_{2}, s_{2}\right) \in \mu$. Therefore, $\left(s_{2}, t_{2}\right) \in \mu$ because $\mu$ is symmetric. Analogously, $\left(s_{2}, t_{2}\right) \in \mu \Rightarrow$ $\left(s_{1}, t_{1}\right) \in \mu$. Lemma 3 is proved.

Consider the quotient set $S^{-} / \sigma$ of all equivalence classes modulo $\sigma$. We define a tolerance space $\left(S^{-} / \sigma, \mu / \sigma\right)$ as follows:

$$
\left(\sigma\left\langle s_{1}\right\rangle, \sigma\left\langle s_{2}\right\rangle\right) \in \mu / \sigma \Leftrightarrow\left(s_{1}, s_{2}\right) \in \mu .
$$

By Lemma 3, this definition does not depend on the choice of representatives $s_{1}$, $s_{2}$ in the equivalence classes $\sigma\left\langle s_{1}\right\rangle$ and $\sigma\left\langle s_{2}\right\rangle$.

By Lemma $2,\left(S^{-} / \sigma, \mu / \sigma\right)$ is isomorphic to an intersection space $(A ; \rho)$. Let $f$ be an isomorphism between the two spaces. For every $s \in S^{-}$define a binary relation $g(s)=f(\sigma\langle s\rangle) \times f\left(\sigma\left\langle s^{-1}\right\rangle\right)$, and let $g(0)=\varnothing$ if 0 is a zero of $S$.

LEMMA 4. The mapping $g$ is an isomorphism of the involuted semigroup $S$ onto an involuted semigroup of constant maps.

Proof. First of all $g(s)$ is a constant map for every $s \in S$. Also, $g$ is one-to-one. Indeed, if $s \in S^{-}$, then $g(s) \neq \varnothing=g(0)$. If $g\left(s_{1}\right)=g\left(s_{2}\right)$ for $s_{1}$, $s_{2} \in S^{-}$, then $f\left(\sigma\left\langle s_{1}\right\rangle\right)=f\left(\sigma\left\langle s_{2}\right\rangle\right)$ and $f\left(\sigma\left\langle s_{1}^{-1}\right\rangle\right)=f\left(\sigma\left\langle s_{2}^{-1}\right\rangle\right)$. Since $f$ is one-to- 
one, we obtain $\sigma\left\langle s_{1}\right\rangle=\sigma\left\langle s_{2}\right\rangle$ and $\sigma\left\langle s_{1}^{-1}\right\rangle=\sigma\left\langle s_{2}^{-1}\right\rangle$, i.e. $\left(s_{1}, s_{2}\right) \in \sigma$ and $\left(s_{1}^{-1}, s_{2}^{-1}\right) \in \sigma$. Therefore $s_{1} s_{1}^{-1}=s_{2} s_{2}^{-1}$ and $s_{1}^{-1} s_{1}=s_{2}^{-1} s_{2}$. By condition (1) of Theorem $4, s_{1}=s_{2}$.

Also, $g\left(s^{-1}\right)=g(s)^{-1}$. Indeed, if $s=0$, then $s^{-1}=0$ and $g\left(s^{-1}\right)=g(0)=\varnothing$ $=\varnothing^{-1}=g(0)^{-1}$. If $s \in S^{-}$, then $g\left(s^{-1}\right)=f\left(\sigma\left\langle s^{-1}\right\rangle\right) \times f(\sigma\langle s\rangle)=g(s)^{-1}$. It remains to prove that

$$
g\left(s_{1} s_{2}\right)=g\left(s_{2}\right) \circ g\left(s_{1}\right)
$$

for all $s_{1}, s_{2} \in S$.

If $s_{1}=0$ or $s_{2}=0$, then both sides of (3) equal $\varnothing$. Suppose that $s_{1}, s_{2} \in S^{-}$. We have

$$
\begin{aligned}
s_{1} s_{2} & \left.\neq 0 \Leftrightarrow\left(s_{1}^{-1}, s_{2}\right) \in \mu \Leftrightarrow\left(\sigma\left\langle s_{1}^{-1}\right\rangle, \sigma\left\langle s_{2}\right\rangle\right) \in \mu / \sigma \Leftrightarrow f\left(\sigma\left\langle s_{1}^{-1}\right\rangle\right) l f\left(\sigma\left\langle s_{2}\right\rangle\right)\right) \\
& \Leftrightarrow \operatorname{pr}_{2} g\left(s_{1}\right) \backslash \operatorname{pr}_{1} g\left(s_{2}\right) \Leftrightarrow g\left(s_{2}\right) \circ g\left(s_{1}\right) \neq \varnothing .
\end{aligned}
$$

Thus, if one side of (3) is $\varnothing$, then (3) holds. Let both sides of (3) be nonempty. Then $s_{1} s_{2} \neq 0$, and $\mathrm{pr}_{2} g\left(s_{1}\right) \backslash \mathrm{pr}_{1} g\left(s_{2}\right)$. Applying condition (3) of Theorem 5, we obtain $s_{1} s_{2}\left(s_{1} s_{2}\right)^{-1}=s_{1} s_{2} s_{2}^{-1} s_{1}^{-1}=s_{1} s_{1}^{-1}$, whence $\left(s_{1} s_{2}, s_{1}\right) \in \sigma$. Analogously, $\left(\left(s_{1} s_{2}\right)^{-1}, s_{2}^{-1}\right) \in \sigma$. Thus $g\left(s_{1} s_{2}\right)=f\left(\sigma\left\langle s_{1} s_{2}\right\rangle\right) \times f\left(\sigma\left\langle\left(s_{1} s_{2}\right)^{-1}\right\rangle\right)=f\left(\sigma\left\langle s_{1}\right\rangle\right) \times$ $f\left(\sigma\left\langle s_{2}^{-1}\right\rangle\right)=\left(f\left(\sigma\left\langle s_{2}\right\rangle\right) \times f\left(\sigma\left\langle s_{2}^{-1}\right\rangle\right)\right) \circ\left(f\left(\sigma\left\langle s_{1}\right\rangle\right) \times f\left(\sigma\left\langle s_{1}^{-1}\right\rangle\right)\right)=g\left(s_{2}\right) \circ g\left(s_{1}\right)$. Thus (3) holds, which completes the proof of Lemma 4 . Theorem 5 follows from Lemma 4.

\section{References}

[1] A. H. Clifford and G. B. Preston, The algebraic theory of semigroups, Volume 1, (American Mathematical Society, Providence, R.I., 1961).

[2] G. Lallement and M. Petrich, 'Décompositions I-matricielles d'un demi-groupe', J. Math. Pures Appl. 45 (1966), 67-117.

[3] W. D. Munn, 'Embedding semigroups in congruence-free semigroups', Semigroup Forum 4 (1972), 46-60.

[4] B. M. Schein, 'Semigroups of rectangular binary relations', Dokl. Akad. Nauk SSSR 165 (1965), 1011-1014 [Russian; for an English translation see: Soviet Math. Dokl. 6 (1965), 1563-1566].

[5] B. M. Schein, 'Relation algebras and function semigroups', Semigroup Forum 1 (1970), 1-62.

[6] B. M. Schein, 'Bands with isomorphic endomorhpism semigroups', J. Algebra (to appear).

[7] A. Tarski, 'Contribution to the theory of models, II,' Nederl. Akad. Wetensch. Proc. Ser. A 57 (1954), 582-588.

\section{Department of Mathematical Sciences}

University of Arkansas

Fayetteville, Arkansas 72701

U.S.A. 